\title{
Reconstruction of Transmembrane Currents Using Support Vector Machines and Its Application to Endocardial Mapping: A Model Study
}

\author{
F Alonso-Atienza ${ }^{1}$, JL Rojo-Álvarez ${ }^{1}$, D Álvarez ${ }^{2}$, M Moscoso $^{2}$, A García-Alberola ${ }^{3}$ \\ ${ }^{1}$ Universidad Rey Juan Carlos, Fuenlabrada, Madrid, Spain \\ ${ }^{2}$ Universidad Carlos III de Madrid, Leganés, Madrid, Spain \\ ${ }^{3}$ Hospital Universitario Virgen de la Arrixaca, Murca, Spain
}

\begin{abstract}
Recent endocardial mapping systems reconstruct an instantaneous image of the endocardial electrical activity performing the inverse problem of electrocardiography (IPE), which consists of estimating the endocardial surface potentials from intracavitary probe potentials. Even though the IPE has been long studied, it still being paid attention due to its ill-posed nature, and many different regularization techniques have been explored in this setting. In this study we analyzed Support Vector machines (SVM) as an alternative regularization technique regarding their robustness against ill-posed problems. We propose here two new SVM algorithms, specifically adapted to the ill-posing issues of the IPE, and develop the equations for endocardial mapping of transmembrane currents. We show, both in simple simulations and in a previously developed cellular automata, that the ill-posing robustness of the SVM is higher when compared to regularized approaches during the depolarization phase. In conclusion, the properties of the developed SVM algorithms stand for an appropriate framework for addressing the IPE.
\end{abstract}

\section{Introduction}

Endocardial mapping is a clinical procedure for studying the electrical activity of the heart, in which an image of the cardiac activation patterns in the endocardium is constructed, and the regions of abnormal electrical behavior can be localized, hence allowing for a proper electrophysiological diagnosis and treatment. Recently, mapping systems are being introduced, which construct an instantaneous image of the endocardial electrical activity by estimating the endocardial potentials from signals measured on a intracardiac non-contact multipole electrode balloon [1]. These techniques emerge from the inverse problem of electrocardiography (IPE) principle, which consists of estimating the endocardial or the epicardial cardiac electrical sources (transmembrane voltage or currents) from a number of probing electrodes. Both epicardial and endocardial potential mapping have been widely studied [2]. However some of its major problems, such as ill-posing, curse of dimensionality, and clinical validation, are still being paid attention. IPE problems are highly ill-posed, mainly due to: (1) the low number of measurements available about the cardiac sources; and (2) the attenuation and spatial smoothing inside the torso (for epicardial imaging) or inside the heart (for endocardial imaging). Intuitively, ill-posedness means that small perturbations of the data result in large perturbation of the solution, leading to oscillatory or non-physiological waveforms. Therefore, regularization techniques are required to stabilize and to constrain the solution, which represent a trade-off between measured data fit and a priori imposed constraints. As a consequence, many regularization techniques have been explored, demonstrating always a limited scope. [3].

In this paper we explore an alternative approach to the IPE, focusing on endocardial mapping applications, based on Support Vector Machines (SVM) [4]. SVM are statistical learning algorithms that emerge from the Empirical Risk Minimization Principle (ERMP), and they have shown excellent performance in many different ill-posed problems [4]. Two of the main advantages of SVM are regularization and robustness against the course of dimensionality, which make it an interesting framework for the IPE requirements. Specifically, we present here the equations of two new SVM algorithms, which are adaptations to the IPE of two recently proposed SVM algorithms for time series interpolation [5].

\section{Forward formulation}

To solve the inverse problem, a formulation to the forward problem is first required, accounting for the relationship between the cardiac volume sources and the volume conductor. In our implementation, cardiac sources are modeled by using a previously developed state machine formulation [6]. Additionally, bioelectrical propagation is simulated according to the monodomain formalism, where 
cardiac tissue has been considered to be an homogeneous, continuous, and isotropic medium, with conductivity $\sigma_{t}$. Under these assumptions, an explicit relationship between transmembrane current $i^{m}$ and transmembrane potential $v^{m}$ can be obtained [7] as:

$$
i^{m}=\sigma_{t} \nabla^{2} v^{m}
$$

We model cardiac tissue a 2D grid sheet lying in the $x y$ plane. Then, the electric potential registered in an external point of the endocardial surface of a volume conductor of homogeneous conductivity $\sigma_{e}$ representing the intracardiac space can be approximated [8] as :

$$
v(p)=\frac{1}{4 \pi \sigma_{e}} \sum_{n} \frac{i_{n}^{m}}{\sqrt{\left(x_{e}-x_{n}\right)^{2}+\left(y_{e}-y_{n}\right)^{2}+z_{e}^{2}}}
$$

where $n$ is an indexation for all the elements of the 2D active tissue, and where the denominator inside the sum term represents the distance from the $n^{\text {th }}$ source element $\left(x_{n}, y_{n}\right)$, to the $e^{t h}$ measurement point $p=\left(x_{e}, y_{e}, z_{e}\right)$.

When considering an array of probing catheters, and by using vector-matrix notation, (2) can be denoted as $\mathbf{v}=\mathbf{H i}$, where $\mathbf{H}(\mathbf{m}, \mathbf{n})=\mathbf{1} / \mathbf{r}_{\mathbf{m n}}$ (with $r_{m n}$ representing the distance between element $m$ to element $n$ ) is a matrix relating the measured potentials array $\mathbf{v}$ to transmembrane currents in the tissue $\mathbf{i}$ at any given time instant. Note that $\mathbf{v}$ is a $[K \times 1]$ vector, with $K$ the number of catheters in the array; $\mathbf{i}$ is a $[L \times 1]$ vector with $\mathbf{i}(k)=i_{k}^{m}$, and matrix $\mathbf{H}$ is $[K \times L]$.

\section{Inverse formulation: SVM algorithms}

As shown in [9], performance of signal processing estimation methods benefits from the application of SVM when properly formulated. We present two SVM algorithms, namely Primal Signal Model (PSM) and Dual Signal Model (DSM), which have been adapted here for endocardial imaging of transmembrane currents. Both algorithms are based on the two algorithms presented in [5] for time series interpolation. Therefore, we skip here many of the details. In order to explore the ill-posedness of the SVM algorithms, we take an ideal situation in which we have the same number of sensors than of current sources, $K=L$. Despite this is an unrealistic situation, we consider that this is the first step to analyze the possibilities of the SVM in IPE. Obviously, the next step, using $K<<L$, is the immediate to be taken, but it is still not considered in this paper.

The PSM algorithm. The signal model in (3), can be expressed as:

$$
v_{k}=\sum_{n=0}^{K-1} i_{n} \cdot h_{n-k}+e_{k}=i_{k} * h_{k}+e_{k}
$$

where $*$ denotes discrete convolution (1D or 2D convolution). We keep the index reference to matrices for notation simplicity of 2D functions. The PSM optimization criterion is given by

$$
J_{P S M}=\frac{1}{2}\|\hat{\mathbf{i}}\|_{2}^{2}+\sum_{k=0}^{K-1} L^{\varepsilon H}\left(e_{k}\right)
$$

where the $\varepsilon$-Huber robust cost is defined in [5] and in [9]. As in common optimization problems, once stated the primal problem, then the dual problem can be obtained, which in this case consists in maximizing:

$$
L_{P S M}=-\frac{1}{2} \eta^{\mathbf{T}}(\mathbf{R}+\delta \mathbf{I}) \eta+\mathbf{v}^{\mathbf{T}} \eta-\varepsilon \mathbf{1}^{\mathbf{T}}\left(\alpha+\alpha^{*}\right)
$$

with respect to the lagrange multipliers $\alpha^{(*)}$, constrained to $0 \leq \alpha^{(*)} \leq C$, where $\eta=\alpha-\alpha^{*}$. Kernel matrix is here given by $\mathbf{R}_{k}=h_{k} * h_{-k}$, which is the autocorrelation matrix of $h_{k}$. Then, the estimate current is given by:

$$
\hat{i}_{k}=h_{k} * \eta_{k}
$$

that defines a regularized problem, which could be limited by the lack of sparseness of the solution current.

The DSM algorithm. In this case, we model observations $\left\{v_{k}\right\}$ as a nonlinear regression of each of the spatial locations $k$, by using a nonlinear transformation $\phi: \mathbb{R} \rightarrow \mathscr{H}$, that maps the locations to an unknown and high dimensional Reproducing Hilbert Kernel Space (RKHS) $\mathscr{H}$. We can build a linear regression model in the RKHS, given by:

$$
v_{k}=\langle\mathbf{w}, \phi(k)\rangle+e_{k}
$$

where $\mathbf{w} \in \mathscr{H}$ is the regression vector. By following the SVM methodology [5], the functional to be minimized is:

$$
J_{D S M}=\frac{1}{2}\|\mathbf{w}\|_{2}^{2}+\sum_{k=0}^{K-1} L^{\varepsilon H}\left(e_{k}\right)
$$

and the dual problem is obtained as the maximization of:

$$
L_{D S M}=-\frac{1}{2} \eta^{\mathbf{T}}(\mathbf{G}+\delta \mathbf{I}) \eta+\mathbf{v}^{\mathbf{T}} \eta-\varepsilon \mathbf{1}^{\mathbf{T}}\left(\alpha+\alpha^{*}\right)
$$

constrained to $0 \leq \alpha^{(*)} \leq C$, and where $\mathbf{G}(j, k)=$ $\langle\phi(\mathbf{j}), \phi(\mathbf{k})\rangle$. Given the convolutional model in (3), it can be shown that

$$
v_{k}=\sum_{j=0}^{K-1} \eta_{j}\langle\phi(j), \phi(k)\rangle=\sum_{j=0}^{K-1} \eta_{j} h_{j-k}
$$

so using the well-known kernel trick [4] (as $h_{k}$ is a Mercer Kernel so $\left.\langle\phi(j), \phi(k)\rangle=h_{j-k}\right)$, the estimated current is given by:

$$
\hat{i}_{k}=\eta_{k}
$$

and then $\hat{v}_{k}=\eta_{k} * h_{k}$. In this case, transmembrane currents are obtained from the Lagrange multipliers, and according to the SVM principles a sparse solution for the currents is achieved, which is a plausible condition for the solution, specially in depolarization conditions. 


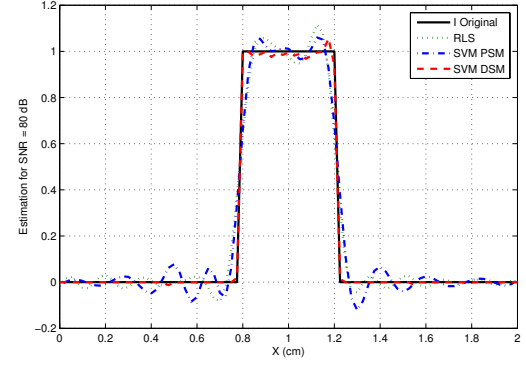

(a)

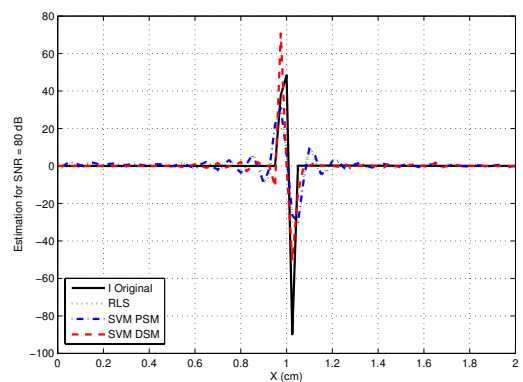

(c)

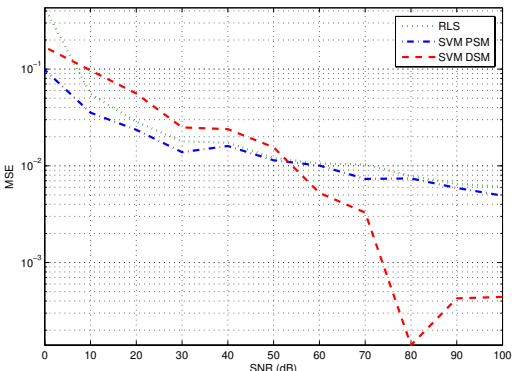

(b)

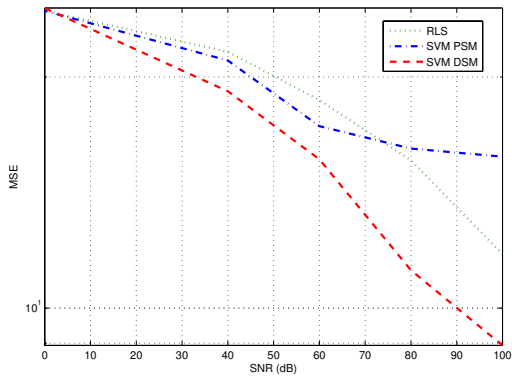

(d)

Figure 1. 1D Simulations. (a) Estimation comparison of RLS, PSM and DSM for a pulse-shape current, (b) algorithms performance vs SNR. (c) Realistic depolarization 1D transmembrane current calculated from an $200 \mathrm{~ms}$ AP travelling at $50 \mathrm{~cm} / \mathrm{s}$ in the $x$ direction. This AP has an spatial length of $\lambda=50 \cdot 0.2=10 \mathrm{~cm}$. For clarifying, just $2 \mathrm{~cm}$ long tissue is depicted, (d) algorithms performance vs SNR.

\section{4. $\quad$ Results}

In this section, PSM and DSM algorithms are compared to a standard Tikhonov approach whose solution is performed by Regularized Least Squares (RLS) method: $\hat{\mathbf{i}}=\left(\mathbf{H}^{\mathbf{T}} \mathbf{H}+\gamma \mathbf{I}\right)^{-1} \mathbf{H}^{\mathbf{T}} \mathbf{v}$, where ${ }^{T}$ and ${ }^{-1}$ denote transpose and inverse of a matrix, respectively, and $\gamma$ is the regularization parameter. A series of experiments have been conducted involving synthetic signals and realistic transmembrane current for both $1 \mathrm{D}$ and $2 \mathrm{D}$ simulations. In all cases, inverse problem has been solved from simulated electrograms corrupted by Gaussian noise. Estimation performance of the transmembrane current has been tested for different Signal to Noise Ratio (SNR) values by means of the MSE criterion: $M S E=\sum_{k=0}^{K-1}\left(i_{k}-\hat{i}_{k}\right)^{2} / K$

$1 D$ Synthetic Data. In this example, a predefined steep current has been chosen to analyzed the regularization properties of the PSM and DSM algorithms. Solving the inverse problem to reconstruct a pulse-shape transmembrane current (Fig.1(a) solid line) demonstrates that the DSM algorithm outperforms PSM and RLS for high SNR values (Fig.1(b)). Indeed, for a $\mathrm{SNR}=80 \mathrm{~dB}$, DSM estimated current fit quite well to the original data. It is also remarkable that the PSM exhibits better performance than the RLS curve in all cases (Fig.1(b)). Inferiority of the
DSM at low SNR is attributed to the SVM free parameters $(\varepsilon, C, \delta)$ tuning procedure, which requires a high computational cost. This hypothesis is corroborated from results to be presented in the following paragraphs.

1D Realistic Data. In this case, transmembrane current has been obtained from (1), where $v^{m}$ is the simulated Action Potential (AP) derived from the automaton model [6]. Fig.1(c) (solid line) represents the depolarization transmembrane current of an AP travelling in the $x$ direction at a fixed time instant. Sparse solution of the DSM algorithm better performs with respect to PSM and RLS for all SNR Fig.1(d), since lagrange multipliers can be well adjusted to counteract abrupt changes in the depolarization current.

$2 D$ Realistic Data. Just the DSM is analyzed here, as 2D image reconstruction is done by using a nonlinear regression of each pixel location. A depolarize transmembrane current 2D image (Fig.2 (a)) is used for studying the estimation properties of the DSM and the RLS algorithms. As expected, DSM outperforms RLS algorithm for almost all SNR, except for very noisy data $(\mathrm{SNR}=0 \mathrm{~dB})($ Fig.2 (b)) even tough both algorithms perform a good estimation. 


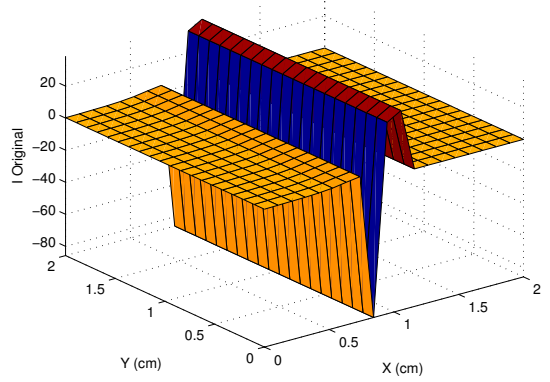

(a)

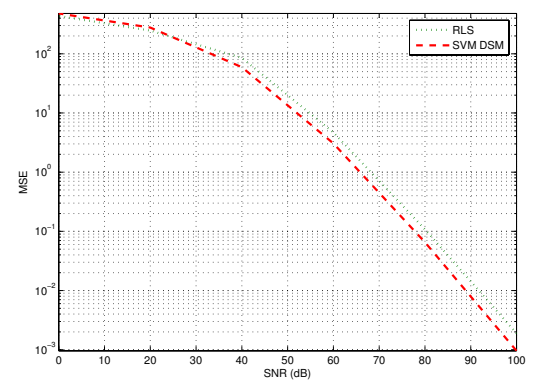

(b)

Figure 2. 2D realistic simulations. Cardiac tissue is represented as a $20 \times 20$ grid sheet lying in the $x y$ plane, with $d x$ and $d y$ having $0.1 \mathrm{~cm}$ resolution. (a) Snapshot of the transmembrane current obtained form the automaton model. (b) Algorithms performance.

\section{Discussion and conclusions}

In the present seminal study, a new regularization approach based on SVM properties has been analyzed. Proposed algorithms, namely PSM and DSM, have shown good performance compared to zero-order Tikhonov regularization. Concretely, DSM sparse solution has demonstrated to be superior in all the cases under study, which confers it a special attention for further analysis. On the other hand, for simplicity, we have found the inverse solution for a single time instant, however the algorithms can be easily extended to include more than one time instant. An important issue of the SVM is the free-parameters tuning procedure which in some cases could reduce SVM performance. This is still an open problem that is paying a lot of attention in the literature. Our results show a promising future research. Firstly, Transmembrane current is an informative magnitude of both depolarization and repolarization processes, and from which activation time patterns can be extracted. This quantities result of great importance to display from the IPE [10]. And second, clinical validation of algorithms presented here can be done by contrasting our results from current mapping systems used in cardiac electrophysiology.

\section{Acknowledgements}

This work was been partially supported by Research Grant from Guidant Spain and by the Research Project TEC2007-68096-CO2/TCM and FIS-2004-03767.

\section{References}

[1] Voth EJ. The inverse problem of electrocardiography: industrial solutions and simulations. IJBEM 2005;7(2):191194.
[2] He B. Modeling and Imaging of Bioelectrical Activity: Principles and Applications. Springer, 2004.

[3] Serinagaoulu Y, Brooks DH, MacLeod RS. Improved performance of bayesian solutions for inverse electrocardiography using multiple information sources. IEEE Trans Biomed Eng 2006;7(10):2024-34.

[4] Vapnik VN. The Nature of Statistical Learning Theory. Springer, 1999.

[5] Rojo-Álvarez JL, Figuera C, Martínz-Cruz CE, CampsValls G, Alonso-Atienza F, Martínez-Ramón M. Nonuniform interpolation of noisy signals using support vector machines. IEEE Trans Sig Process ;55(8):4116.

[6] Alonso-Atienza F, Requena-Carrión J, García-Alberola A, Rojo-Álvarez J, Sánchez-Muñoz J, Sánchez JM, ValdésChávarri M. A probabilistic model of cardiac electrical activity based on a cellular automata system. Rev Esp Cardiol 2005;58(1):41-47.

[7] Malmivuo J, Plonsey R. Bioelectromagnetism: Principles and applications of Bioelectric and Biomagnetic Fields. Oxford University Press, 1995.

[8] Spach MS, 3rd WTM, Miller-Jones E, Warren RB, Barr RC. Extracellular potentials related to intracellular action potentials during impulse conduction in anisotropic canine cardiac muscle. Circ Res 1979;45(2):188-204.

[9] Camps-Valls G, Rojo-Álvarez JL, Martínez-Ramón M. Kernel methods in bioengineering, communications and image processing. Idea Group Inc., 2006.

[10] MacLeod R, Kornreich F, van Oosterom A, Rautaharju P, Selvester R, Wagner G, Zywietz C. Report of the first virtual visualization of the reconstructed electrocardiographic display symposium. J Electrocardiol 2005;38(4):385-99.

Address for correspondence:

José Luis Rojo Álvarez

B004, Universidad Rey Juan Carlos, Camino del Molino s/n, 28943 Fuenlabrada, Madrid, Spain. Email: joseluis.rojo@urjc.es 